\title{
EDITORIALES Y EDITORAS EN BRASIL HOY. DOS CASOS CONTEMPORÁNEOS: CHÃO DA FEIRA Y RELICÁRIO ${ }^{1}$
}

\author{
ANA ELISA RiBEIRO \\ Centro Federal de Educação Tecnológica de Minas Gerais
}

A partir de la constatación de que son escasas o inexistentes las narrativas históricas de mujeres editoras en Brasil, nuestro objetivo es hacer un registro, tan completo como sea posible, de la historia editorial y de la actuación de las editoras en el país, especialmente en los siglos XX y XXI, a partir de dos estudios de caso. En este artículo, mostramos un primer elenco de personajes relevantes tanto para la edición brasileña actual como para la historia de la ocupación del espacio público por parte de la mujer contemporánea. Más concretamente, se presentan dos ejemplos de las editoriales dirigidas por mujeres que trabajan en la escena literaria contemporánea: las representantes de las editoriales Chão da Feira (Belo Horizonte/Lisboa/São Paulo) y Relicário (Belo Horizonte, Minas Gerais).

PALABRAS CLAVE: mujeres editoras, producción editorial, historia editorial, Brasil, edición brasileña.

Publishing Houses and Women Publishers in Brazil Today. Two Contemporary Case Studies: Relicário and Chão da Feira

Since the historical narratives of women publishers in Brazil are scarce or non-existent, our goal in this work is to record, as completely as possible, the editorial history and the work of women publishers in the country, especially in the 20th and 21st centuries, beginning with two case studies. In this paper, we present a set of relevant characters both to understand Brazilian publishing practices and to sketch the history of the occupation of public spaces by contemporary women. More in particular, we present two examples of publishing houses directed by women who work in the contemporary Brazilian literary scene: the publishers of Chão da Feira (Belo Horizonte/Lisbon/São Paulo) and Relicário (Belo Horizonte, Minas Gerais).

KEY WORDS: women publishers, editorial production, editorial history, Brazil, Brazilian edition.

Este trabajo es parte de una investigación mayor titulada Mujeres que editan: Un mapa preliminar en Brasil, desarrollada desde 2016 en el Programa de Postgrado

\footnotetext{
${ }^{1}$ Agradecemos al CNPq, a la Fapemig y al CEFET-MG por la ayuda recibida para la presentación previa de este trabajo en el 56 Congreso de Americanistas, en Salamanca, 2018. Gracias a Alejandra Torres Torres y Sérgio Karam por la lectura final de este artículo.
} anadigitalpro@gmail.com 
en Estudios de Lenguajes del Centro Federal de Educação Tecnológica de Minas Gerais. A partir de la constatación de que son escasas o inexistentes las narrativas históricas de editoras brasileñas en la bibliografía de Brasil, nuestro objetivo es registrar, de la forma más completa posible, la historia profesional de estas mujeres en el país, en los siglos XX y XXI, desde su invisibilidad total al protagonismo adquirido en la producción editorial y literaria.

A pesar de la existencia de estudios aislados sobre la historia editorial de Brasil, las menciones de las editoras literarias, en especial en el sector destinado a los lectores adultos, solo empiezan a aparecer a partir de 1980, como puede comprobarse en la obra de referencia O livro no Brasil, de Laurence Hallewell (2005). ${ }^{2}$ Si bien el sector editorial es un campo típicamente masculino, las editoras también han dejado su impronta en él, sobre todo desde finales del siglo XX, aunque falte la narrativa que reivindique sus logros. Como señala la socióloga de la literatura Gisèle Sapiro (2016), la perspectiva de género es necesaria en los estudios sobre la edición, punto de partida de este trabajo.

En este panorama tan reciente, creemos encontrar elementos para una narrativa más justa y más precisa de la actuación de los editores en Brasil, tanto hombres como mujeres, entre los agentes de la producción editorial y literaria contemporánea. Por citar algunos ejemplos que faltan o cuya actividad no se ha resaltado de forma conveniente en trabajos como los de Hallewell (2005), podemos recurrir a los casos de Zahidé Lupinacci Muzart (Cruz Alta, 1937-Florianópolis, 2015), de la editorial Mulheres, apenas mencionada, y de Maria Mazarello Rodrigues (Ponte Nova, 1941-), fundadora de la Mazza Edições y simplemente no citada. Muzart y Rodrigues son dos editoras de extrema importancia para la expansión y diversificación del mercado literario brasileño fuera del eje Rio de Janeiro-São Paulo (Pereira, Freitas, Fonseca y Santana, 2015). La primera es fundadora de una editorial especializada en libros feministas y en autoras olvidadas del siglo XIX, con un catálogo de más de cien títulos publicados desde 1995 en la ciudad de Florianópolis; ${ }^{3}$ y la segunda, Maria Mazarello Rodrigues, de un sello creado en Belo Horizonte en 1981 para difundir a autores negros de Brasil y de otros entornos geográficos.

A fin de contribuir a una narrativa sobre la edición en Brasil que supere una visión centralizadora ligada al eje Rio de Janeiro-São Paulo, escogemos dos casos de sellos dirigidos por mujeres, cuyas actuaciones vienen obteniendo visibilidad y

\footnotetext{
${ }^{2}$ La obra de Hallewell fue originalmente publicada en el año de 1985, con nuevas ediciones en 2005 y 2012 en la editorial de la Universidade de São Paulo. La bibliografía sobre edición de libros en Brasil es relativamente reciente y necesita ampliarse (Ribeiro, 2017).

${ }^{3}$ Junto con Sérgio Karam, investigador de la Universidade Federal do Rio Grande do Sul, estoy trabajando en el estudio del catálogo de la editorial Mulheres y de su editora principal, la profesora Zahidé Muzart.
} 
reconocimiento entre sus pares más allá de sus estados - e incluso del país- de residencia. Se trata de Relicário (2013), de la editora Maíra Nassif, y de Chão da Feira (2011), de las editoras Carolina Fenati y Luisa Rabello, Júlia de Carvalho Hansen y Cecília Rocha. En ambos casos, las empresas se fundan en la ciudad de Belo Horizonte, capital de Minas Gerais, desde donde operan, si bien el aporte de las tecnologías digitales ofrece a este tipo de proyectos una dimensión mucho más amplia y colectiva.

Para recabar datos e informaciones en torno a Relicário y Chão da Feira, además de recurrir a los sitios web de las editoriales, solicitamos una entrevista por escrito a las responsables de los sellos, así como intercambiamos varios mensajes añadidos. Es nuestro objetivo, por lo tanto, constituir una narrativa sobre sus modos de actuar, tomando como modelo las configuraciones del campo según John Thompson (2013), especialmente cuando trata de la economía de "favores" en la que operan las editoriales pequeñas como sus antecesoras Mulheres y Mazza.

\section{Pequeñas editoriales: un modelo de actuación}

En el capítulo "A polarização do campo" de la obra Mercadores de cultura, el sociólogo John B. Thompson (2013) se ocupa del estudio de la escala de las editoriales, actualmente divididas entre las enormes, resultado de la fusión de grupos multinacionales, y las pequeñas y medianas, que intentan sobrevivir en un escenario muy saturado. ¿Qué es y cómo funciona una pequeña editorial en el siglo XXI? En este sentido, la principal pregunta que anima el trabajo de Thompson es: “¿Por qué hay tantas pequeñas operaciones editoriales en el sector de la publicación de libros?". ${ }^{4}$ La respuesta se centra en elementos como el bajo costo que implica promover una empresa en el sector; la revolución digital; la fácil externalización de partes del proceso de edición; el abaratamiento de los costos en algunas fases de la producción, así como lo que Thompson llama la "economía de favores". Para el autor, "esos mundos [el de las multinacionales y el de las pequeñas editoriales] no se superponen, porque la brecha entre ellos, en términos de la escala de recursos que tienen a su disposición, es simplemente demasiado grande" (2013: 170-171). Así, la "economía de favores" sería el intercambio de competencias, conocimientos y contactos entre las pequeñas editoriales:

Ellas [las editoriales pequeñas] se ven como parte de una vocación común y una misión compartida. Sus rivalidades competitivas son ofuscadas por las afinidades que provienen de sus propósitos comunes, de su comprensión compartida de las dificultades enfrentadas por todas las pequeñas

\footnotetext{
${ }^{4}$ Las traducciones al español son de mi autoría.
} 
editoriales y de su oposición colectiva al mundo de las grandes editoriales corporativas. (Thompson, 2013: 171)

Otros elementos que hacen viables los proyectos pequeños estarían incluidos entre las prácticas de la "economía de favores": costos más bajos por tareas realizadas por freelancers, trabajo del propio editor, relación amistosa con librerías y puntos de venta también “independientes”, entre otros. Según el sociólogo, las editoriales pequeñas establecen una fuerte relación pasional por el mundo del libro, y normalmente son representadas por el propio editor o editora fundador.

Thompson alerta sobre aspectos como la subcapitalización de las pequeñas operaciones, que mantiene siempre en apuros financieros a estos sellos pequeños; las dificultades para entrar en el "radar" de los grandes medios de comunicación y la dependencia que algunas de las empresas tienen con uno o dos títulos de éxito en su catálogo. A esta situación, se le suma la pérdida de autores que pasan a grupos más grandes y los acuerdos difíciles para la distribución y las ventas.

En los dos casos aquí focalizados, es posible identificar elementos de esa "economía", pero estos nos parecen más ejemplos de colaboración e incluso de "intercambio" que propiamente de "favores". Los pequeños sellos Relicário y Chão da Feira emergen en la década del 2000, de la pasión por los libros y del deseo de traducir buenos textos para el lector en lengua portuguesa y, al mismo tiempo, de una actividad en red, inspirada en el modo de actuación de otras editoriales de pequeñas dimensiones, entrenadas en el cuidado de correr riesgos bien calculados.

\section{Dos casos notables de pequeñas editoriales contemporáneas}

Así, a partir de los intercambios de correo electrónico entre los meses de febrero y mayo de $2018^{5}$ con Maíra Nassif, editora de Relicário, y con Júlia de Carvalho Hansen y Carolina Fenati, dos de las cuatro fundadoras de Chão da Feira, reconstruiremos la historia, la gestión y la visión de estas mujeres en relación con sus sellos y con el escenario editorial del momento.

\section{Chão da Feira: gatos, hijos, perros y libros alrededor}

La editorial Chão da Feira - o CF- fue fundada en diciembre de 2011, a partir de la convocatoria de Carolina Fenati a colegas para la composición de un producto editorial llamado Caderno de Leituras. El núcleo fundador de la casa editorial lo integraban la propia Fenati, Júlia de Carvalho Hansen, Cecília Rocha y Luisa

\footnotetext{
${ }^{5}$ Las editoras prefirieron que las entrevistas fueran por escrito, a fin de elaborar mejor sus respuestas. Algunas conversaciones informales también han sido importantes para la elaboración de este trabajo.
}

230

Lectora, 25 (2019): 227-240. ISSN: 1136-5781 D.O.I.: 10.1344/Lectora2019.25.14 
Rabello, grupo que sigue trabajando en la actualidad. Las editoras se conocieron en el contexto académico, mientras Fenati cursaba el doctorado en Lisboa, donde estudiaba también Júlia Hansen, y Chão da Feira es el nombre de la calle donde vivía la primera en Lisboa. Según Júlia Hansen, se trata de una denominación que "trae simultáneamente una idea de durabilidad y de sencillez"6 o, según Fenati, "una idea de simplicidad, y al mismo tiempo de posicionamiento en el compartir lo sensible: estamos siempre fuera de cualquier cosa que se instituye como centro, sea de poder, de legitimidad, de autoría, etc., y buscamos afirmar lugares 'para todos y para nadie"”.

Según Júlia Hansen, Chão da Feira es "un colectivo de editoras que adoran trabajar juntas y hacer bien las cosas", lo que apunta a las relaciones entre la producción editorial y la materialidad de los libros publicados; la elección de los textos; la relación entre las editoras, los autores y todos los profesionales involucrados en el sello. Carolina Fenati complementa la visión de la editorial: "Quiero un catálogo de libros que inspiren para la vida”. Y la fundadora ofrece un ejemplo:

publicamos un libro titulado $O$ pobre de pedir. Es el último libro de Raul Brandão, y una meditación o peregrinación sobre experiencias difíciles: frustación, miseria, mezquindad, muerte. [...] Para quien lee, este libro es un modo de observar estas experiencias, y por lo tanto, en alguna medida, vivirlas sin ser devorados por ellas, y con un aliento que solo un maestro en la lengua puede tener. Es esto lo que entiendo como un libro que intensifica las relaciones entre literatura y vida.

Carolina Fenati, Júlia Hansen, Luisa Rabello y Cecília Rocha comparten un mismo ideal en su empresa editora, si bien el perfil de cada una de ellas es muy diverso en cuanto a su formación y experiencias. Carolina Fenati (1982) nació en Belo Horizonte, se graduó en Historia por la Universidade Federal de Minas Gerais, es doctora en Literatura Inglesa y estudió en Lisboa, pero se gana la vida como terapeuta; Júlia Hansen (1984), formada en Letras por la Universidade de São Paulo, en su ciudad natal, trabaja como astróloga; Cecília Rocha (1981), procedente de Goianésia (Goiás), vive en Belo Horizonte, tiene formación en Comunicación Social (UFMG) y trabaja en el equipo de curaduría del museo de arte de Inhotim; y, por último, Luisa Rabello (1985), de Belo Horizonte, es la artista gráfica de Chão da Feira, si bien también se cuenta con la participación de otros profesionales.

Como se ha indicado, la iniciativa empezó con la publicación de los primeros números de Cadernos de Leituras y siguió con libros y la revista Gratuita. Según

\footnotetext{
${ }^{6}$ De aquí en adelante, todas las citas no identificadas provienen de las entrevistas realizadas.
} 
Júlia Hansen, los textos suelen llegar por una invitación previa a autores y traductores, quienes tienen una fuerte participación en el proceso editorial. Según Carolina Fenati: "Casi siempre es en esta intersección entre el deseo de los autores y la propuesta de la Chão donde germinan los libros”. Júlia Hansen describe el proceso editorial de $\mathrm{CF}$, marcado por el carácter independiente, múltiple y colectivo:

Actuamos juntas, pero cada una hace una cosa diferente conforme a su posibilidad y especialidad y en general intervenimos en todo, en cada detalle; de la flor que llevamos a la mesa de nuestros libros en una feria de libros, al papel escogido para una publicación. No somos socias en sentido jurídico. Hay varias divisiones de los trabajos; por ejemplo, Luisa es la editora de arte de Chão da Feira; cada una de nosotras, en algún momento, ha sido la responsable de llevar los pedidos al correo; Carolina es la editora de Chão da Feira, yo soy editora adjunta [...]. Y creo que eso es lo que hace que disfrutemos tanto de trabajar juntas. Ahora, tenemos un pasante y algunos colaboradores que se ocupan desde la asesoría de prensa hasta las correcciones y los trabajos gráficos.

Y Carolina Fenati completa:

Chão da Feira es una editora independiente, y esto implica un funcionamiento muy específico, para el cual no hay modelos. Una editorial independiente no funciona como una gran editorial, no es una versión en miniatura de una empresa. Como colectivo, buscamos crear formas de trabajo que respeten, por ejemplo, el tiempo de cada una, y en el que el ritmo de producción no nos haga perder ni el cuidado ni el placer de trabajar. ¿Es difícil? Lo es. También es muy gratificante.

En cuanto a aspectos formales, Chão da Feira se constituye como empresa registrada en el Cadastro Nacional de Pessoas Jurídicas (CNPJ) ${ }^{7}$ y define los costos del material que publica con mucha cautela, sin aporte de los autores y traductores. Los nuevos libros se contratan a medida que los anteriores se pagan. La CF no tiene una relación estrecha con librerías, pues mantiene como principales canales de ventas su sitio web y las ferias de libros independientes. Es en estos espacios donde los libros encuentran su mayor flujo, si bien las editoras manifiestan el deseo de expandir su relación con los libreros y mejorar la distribución de sus publicaciones.

\footnotetext{
${ }^{7}$ Cadastro que registra y regulariza las empresas, dándoles el estatus de empresas efectivas, oficiales, con implicaciones para su reconocimiento oficial y su fiscalización.
} 
Chão da Feira se incluye en un marco más amplio de pequeñas editoriales en Brasil, participa de eventos en el país, pero no está afiliada a asociaciones profesionales de editores. El mismo cuidado que las fundadoras tienen con el proceso editorial está explícito en la construcción de su catálogo. Hasta marzo de 2018, estaba compuesto por 14 libros, 3 números de la revista Gratuita — de circulación gratuita y tirada de miles de ejemplares- y 74 de Caderno de Leituras, publicados en línea en la web del sello editorial: ${ }^{8}$

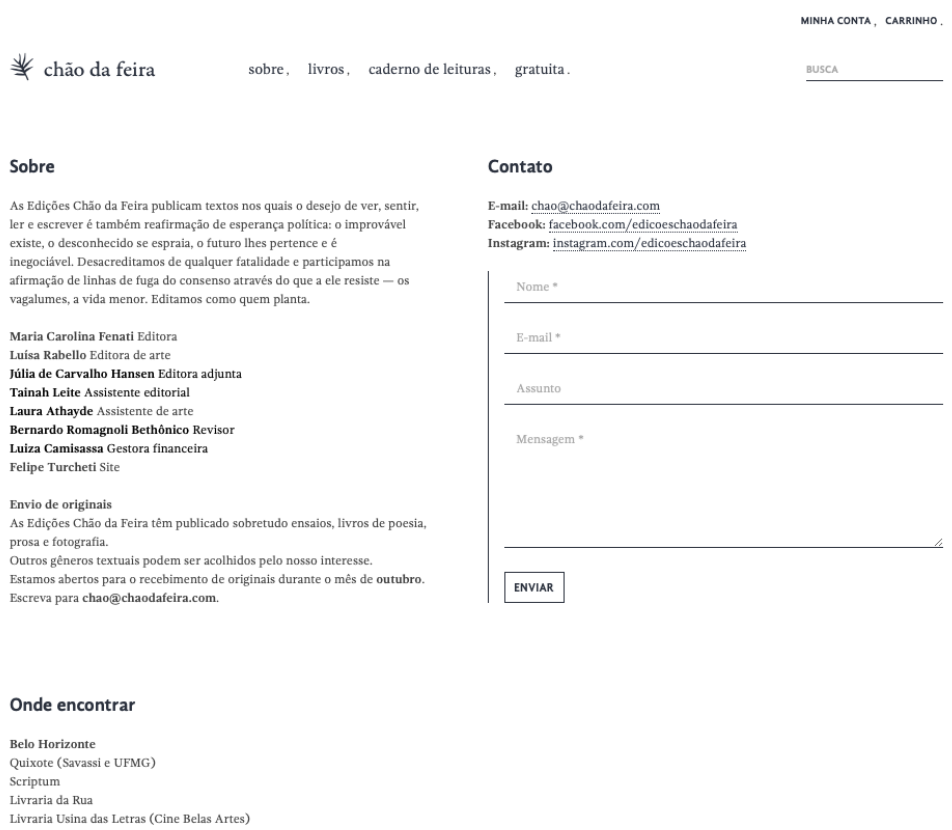

Fig. 1: Página web de la editorial Chão da Feira. Fuente: Printscreen de $<$ http://chaodafeira.com/sobre/>. Fecha de consulta: 08/07/2019

En el marco de una red contemporánea de editoriales, Chão da Feira reconoce otros sellos de perfil similar al suyo y en los que se inspira. Júlia Hansen y Carolina Fenati mencionan Relicário, de Belo Horizonte, y Zazie, de Rio de Janeiro, con las que mantienen relaciones de colaboración. Las portuguesas Assírio e Alvim, \&Etc, Hiena, Cotovia y Relógio D’Água son también fuente de inspiración. Según Fenati, "en Brasil, la profusión de pequeñas empresas editoras es ya un impulso para continuar”, entre las cuales menciona Piseagrama y Não-Edições.

\footnotetext{
${ }^{8}$ En la programación de 2018, la estimación de las editoras era publicar al menos tres nuevos libros, en su mayoría traducciones.
} 
A pesar de encontrar dificultades para la sostenibilidad de CF, las editoras consideran un gran placer emprender la publicación de los libros que creen relevantes para su catálogo y para la vida, cuyo futuro avistan con optimismo, con "muchos libros, algunos de los cuales hoy no podemos ni siquiera imaginar que existen, un espacio para hacerlos con cuidado y osadía, nosotras muy muy amigas, y lo que venga”, como señala Carolina Fenati; “Trabajando juntas, con gatos, hijos, perros y libros alrededor", apostilla Júlia Hansen.

\section{Relicário: la mirada total}

Relicário Edições fue fundada en 2013 por Maíra Nassif (Belo Horizonte, 1985), con el objetivo de publicar libros de interés de la propietaria, graduada y maestra en Filosofía por la Universidade Federal de Minas Gerais y ex productora editorial en una empresa donde descubrió su gusto por la edición de libros. Para fundar Relicário, se registró como microemprendedora individual (MEI, que luego evolucionó a Microempresa), lo que implica un número en el Cadastro Nacional de Pessoas Jurídicas (CNPJ); ${ }^{9}$ acto seguido, envió correos electrónicos a una lista de contactos para dar noticia de la apertura de la editorial y para incitar a los interesados a publicar buenas obras.

Para Maíra Nassif, Relicário es la concreción de un deseo al mismo tiempo íntimo y político: "Creo en la idea de que los libros conectan tiempos y espacios, crean comunidades y ensanchan nuestra experiencia, proponiendo nuevos campos de lo posible". Así, afirma no buscar la innovación, sino hacer, "de la mejor forma posible, ese objeto que es producto material y de contenido, de espíritu y materialidad". La casa editorial nació en un período de crisis económica en Brasil y las condiciones generales se caracterizaron por el número reducido de lectores brasileños y los problemas estructurales en las áreas de la educación y de acceso a los bienes culturales. La editora es consciente de que se dirige a un público específico, lo que transforma las dificultades del campo en una actitud más cautelosa a la hora de tomar decisiones.

En el sitio web de la editorial, el nombre Relicário es explicado como una "caixa ou baú onde se guardam objetos de grande valor afetivo". Y Nassif complementa la definición: "La verdad es que hace tiempo las palabras poseen una morada cautiva; el libro es por excelencia el Relicário de las letras".

Relicário Edições, afiliada a la Liga Brasileira de Editoras (LIBRE), se sitúa en el tradicional barrio Floresta, en Belo Horizonte, en la casa en que Nassif reside. El

\footnotetext{
${ }^{9}$ El MEI (Microempreendor individual, en portugués) es una de las figuras jurídicas existentes en Brasil para regularizar empresas de una sola persona. Tiene implicaciones en lo relativo al pago de impuestos y permite la posibilidad de jubilación del emprendedor.
}

234

Lectora, 25 (2019): 227-240. ISSN: 1136-5781 D.O.I.: 10.1344/Lectora2019.25.14 
hecho de tener la editorial fuera del eje Rio-São Paulo conlleva algunas dificultades añadidas, tales como una divulgación más lenta a escala nacional. Para facilitar esto, Relicário trabaja con asesoría de prensa y la propia editorial negocia directamente, en Belo Horizonte, con libreros y espacios donde exponer los libros, presentes en ferias, "de las menores y más independientes a las mayores y ya consolidadas". Según Maíra Nassif, todavía no es posible participar en bienales y en ferias internacionales debido al alto costo de los stands. Sin embargo, lamentando esa imposibilidad, señala: "nos gusta mucho participar en Ferias para tener contacto directo con los lectores".

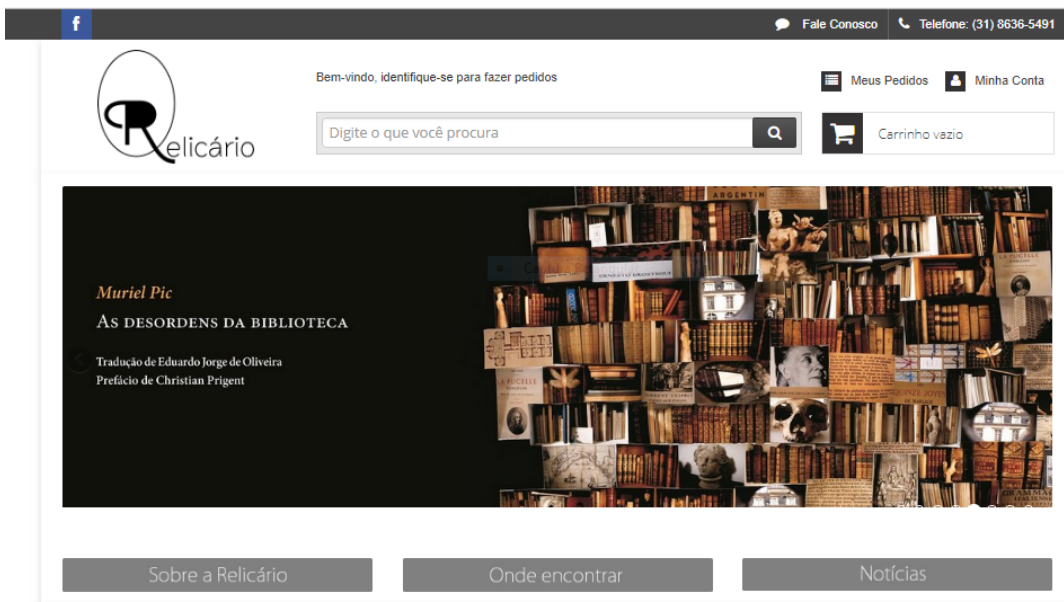

Fig. 2: Página inicial del sitio web de Relicário Edições. Fuente: Printscreen de <https://Relicárioedicoes.loiaintegrada.com.br/>. Fecha de consulta: 29/03/2018.

La primera obra publicada por Relicário fue Personagens conceituais. Filosofia e arte em Deleuze, de Fernando Tôrres Pacheco, con quien la editora compartió los costos de producción. La obra era un buen ejemplo de la línea editorial que deseaba cultivar. Más adelante la amplió centrándose de un lado en libros teóricos, especialmente de filosofía, estética y crítica literaria, y de otro en literatura, focalizada en autores nacionales, fundamentalmente contemporáneos, y en traducciones de autores extranjeros poco conocidos en Brasil.

Los libros teóricos generalmente llegan a su conocimiento por medio del envío de originales por parte de los autores, y para la selección de las obras cuenta con la ayuda de un consejo de académicos. En el 20\% de los casos, sin embargo, estos libros son proyectos de la propia editorial. Se trata de obras como Antiga musa (de 
Jacyntho Lins Brandão) y de la Coleção Estéticas, que cuenta con traducciones como Literatura de esquerda, del argentino Damián Tabarovsky.

Los originales de literatura brasileña suelen llegar a la editorial presentados por los propios autores o encargados por Nassif. En cuanto a los extranjeros, los amigos y los componentes del comité editorial de Relicário sugieren traducciones. Más recientemente, Nassif consideró un gran acierto haber invertido en su catálogo literario, citando obras como Jamais o fogo nunca (Jamás el fuego nunca), de la escritora chilena Diamela Eltit, que supuso la proyección de Relicário a nivel nacional con la invitación a la Festa Literária de Paraty (Flip), en 2017, lo que dio visibilidad a la editorial en los medios de prensa y en las librerías. A principios de 2018, Relicário contaba con cuarenta obras en su catálogo, lo que supone una media de diez lanzamientos al año. Estaba prevista la producción de otros veinticuatro libros, entre ellos una caja con dos volúmenes de la poeta argentina Alejandra Pizarnik (Os trabalhos e as noites y Árvore de Diana). ${ }^{10}$

Es una preocupación constante de Maíra Nassif preservar y construir la identidad de su catálogo, dando preferencia a "una línea editorial pequeña, pero consistente". Así, prefiere autores y autoras "ya con cierto equipaje y reconocimiento", y explicita:

[N]o me interesa promover nuevos escritores en el inicio de su carrera, sino invertir en el apoyo mutuo entre editora y autor(a); es decir, publicarlos será bueno no solo para el autor, sino también para el catálogo de la editorial, que ganará aún más consistencia con su presencia. Otro objetivo es el de no rendirme a las publicaciones pagadas. Aquí no basta con pagar para ser publicado. Es necesario tener calidad.

Maíra Nassif destaca que el proceso de producción de los libros de Relicário se desarrolla en intenso y efectivo diálogo con los autores, prácticamente en todas las etapas: corrección, aprobación, diseño gráfico, divulgación, etc. Para ella, esa es la "ventaja de las pequeñas editoriales, pues cuanto menor es la estructura, menores son las mediaciones internas". Esto propicia más proximidad entre autores y productores, así como una mejor comunicación entre todos, lo que trae resultados más satisfactorios: "Jamás continuamos con una cubierta que no ha sido aprobada y deseada por el autor, lo que sucede en otras editoriales que se consideran dueñas de todo el proceso, relegando al autor a un papel secundario".

El costo de los libros de Relicário obedece a un cálculo que toma en consideración el pago de los profesionales involucrados en la producción editorial. Para

\footnotetext{
${ }^{10}$ El lanzamiento, en agosto de 2018, tuvo mucho éxito y logró ocupar las portadas de los suplementos culturales de los periódicos brasileños.
} 
obtener algún beneficio, se cuenta, en algunos casos, con subvención, como sucede con los libros académicos, pues su venta es dificultosa; no sucede lo mismo con las publicaciones literarias, que la editorial financia al completo, pues considera que ese tipo de obra tiene "retorno un poco más garantizado, aunque a menudo con cierta lentitud".

Maíra Nassif actúa sola en el trabajo de administración y en las decisiones editoriales; no tiene empleados contratados, pero cuenta con una red de profesionales freelancers. Ella misma hace la revisión de las pruebas de los libros, pues afirma: "No puedo concebir la idea de poner en el mundo un libro con la marca Relicário sin que haya pasado por mi mirada". En cuanto a la distribución, la editora declara su obstinación y persistencia en ver los volúmenes al alcance del público lector por lo que invierte en una tienda virtual, pero no únicamente; así, creó lo que llama "algunas líneas de fuga del sistema hegemónico de distribución y divulgación de libros", tales como la participación en ferias independientes de publicaciones, la divulgación por Facebook y por mailing. Sin embargo, subraya:

Yo, sinceramente, creo mucho en el encuentro casual y epifánico que hay entre los libros en las estanterías y los lectores que frecuentan las librerías. Digo esto por mi propia experiencia, descubriendo muchos títulos que no tendría la oportunidad de conocer si no fuera por esa contingencia del encuentro en aquel tiempo-espacio. De ese modo, hago constantemente contacto con librerías y distribuidores para ampliar el alcance del libro y el encuentro con los lectores.

Maíra Nassif dice conocer a muchas otras mujeres en la función de editoras: "tengo la impresión de que me relaciono más con mujeres editoras que con hombres editores. No hay una red de colaboración formal, pero siento que sí hay una búsqueda de intercambio de experiencias, diálogo y la disponibilidad de ayudarnos unas a otras". En relación al contexto actual de efervescencia de editoriales pequeñas, explicita:

Creo que estamos viviendo un momento en que todas las pequeñas editoriales se han inspirado e influenciado mutuamente. Muchas haciendo un trabajo increíble de investigación y curaduría, trayendo a nuestro mercado autores y autoras que las grandes editoriales no publican, apostando por diseños gráficos audaces e incluso con procedimientos más artesanales.

Maíra Nassif vive exclusivamente de sus diez horas de trabajo diario en el sello Relicário, después de dos años conciliando con una actividad de productora de contenidos para Internet. Aprendió a editar sin cursos especializados o formación 
institucionalizada, consciente de que su experiencia académica y de buena lectora, además de su "espíritu práctico", la dirigen bien en la realización de sus actividades.

Según la dueña de Relicário Edições, la recepción de los libros que edita es cada vez mejor: "un libro abre las puertas al prójimo". Esto se observa en las entradas espontáneas de lectores en las redes sociales y en las reseñas escritas por críticos. Nassif narra que es gratificante experimentar en las ferias cómo los lectores se acercan a su tienda y dicen: "ya tengo ese, ese, ese, ese... ese conozco ese y ese". El reconocimiento positivo hace pensar en un futuro de crecimiento, aunque ella afirme no renunciar a la proximidad con todo el proceso editorial. "Jamás seré solo una publisher", enfatiza: "la mejor propaganda que una editorial puede hacer son sus propios libros".

\section{Las pequeñas - pero importantes- editoriales y algunas conside- raciones}

Relicário y Chão da Feira son dos editoriales pequeñas y periféricas geográficamente respecto al núcleo que suponen Rio de Janeiro y São Paulo. En el tamaño y en su ethos de editoriales que buscan calidad, cuidado y una elegancia simple en sus proyectos gráficos, ambos sellos pueden ser considerados similares; también en el hecho de que han sido fundados y gestionados por mujeres. Sin embargo, hay un aspecto que diferencia ambos proyectos, al menos en lo tocante a cómo hacen la gestión del negocio, pequeño, pero negocio al fin: mientras Relicário se confunde con la voz de su propietaria, Chão da Feira es una experiencia coral de cuatro mujeres, preocupadas por reconocerse como un colectivo que todo lo negocia y decide en conjunto.

El discurso de la calidad, de la sintonía con un lector cercano, del cuidado de la relación con los autores y las traducciones, de la búsqueda de obras olvidadas o poco visibles, de los ritmos más lentos, del trabajo directo con proveedores y autores, muestran la existencia de un modo casi artesanal y personal de editar. La pequeña editorial existe hoy porque hay oportunidades y situaciones propicias, tanto a nivel ideológico como tecnológico y económico, que la hacen no solo un negocio posible, sino también viable a largo plazo, aunque dependiente de la persona que lo funda; pero también porque hay un modo de hacer libros que actúa como una fuerza contraria a la estandarización o uniformización total, al aplanamiento o a la insipidez de las posibilidades del texto, del discurso, de la literatura o el diseño gráfico. Los pequeños sellos que se identifican con los dos aquí tratados ofrecen incluso un refuerzo importante a la existencia del impreso, ya que miran con cuidado especial las cuestiones ligadas a la materialidad del libro, preocupándose por la elaboración de proyectos gráficos potentes, muchas veces con elementos manufacturados o artesanales. 
Desde el punto de vista del flujo de los libros producidos, ambas editoriales usan Internet como vitrina y tienda, además de hacerse visibles en las muchas ferias hoy existentes. La desigualdad reside en el hecho de que Relicário de Maíra Nassif parece invertir más energía en la relación con las librerías, un modo de distribución tradicional y problemático, desde el punto de vista de los costos, para una pequeña editorial con limitaciones financieras. Es, sin embargo, posible afirmar que, para autores y lectores, la librería es un punto favorecedor de la relación con el libro, incluyendo allí su legitimación y su visibilidad.

Aunque Thompson trata de las pequeñas editoriales como parte de una economía de "favores", considero preferible pensar en fórmulas de colaboración y de solidaridad, con mucho profesionalismo, ya que unas editoriales se inspiran en otras de igual tamaño y con los mismos objetivos, las cuales buscan crear, cada una, un perfil y un discurso que, a la vez, las aproxima y las distingue. Son elementos de ese discurso - erigido para la propia existencia de la editorial y su circulación- las cualidades literaria y gráfica, así como el cuidado en la composición de catálogos cortos, pero relevantes, por medio de una selección de obras elegidas con criterio.

Mientras el nombre de Relicário y el de su propietaria-editora son casi sinónimos, Chão da Feira es un colectivo. La editorial luso-brasileña funciona a ritmo más lento, produciendo casi únicamente para la subsistencia, con decisiones compartidas - incluso para la entrevista que me fue concedida, cuando Júlia Hansen y Carolina Fenati fueron portavoces del grupo, siempre con respuestas con copias para todas las componentes del equipo. Relicário demuestra un modus operandi más convencional, desde el punto de vista de la producción editorial y del flujo de trabajo, aunque explicite bien su idea de la composición de un catálogo cuidado directamente por la propia fundadora. Como pequeña empresa, conoce las inversiones posibles y los riesgos asumidos, cumpliendo lo que distingue a una micro o pequeña editora: aventurarse en la novedad, en la diversidad y en el contraste con el mainstream.

El factor de género, en ambas editoriales, comparado con otras editoriales dirigidas por hombres, igualmente jóvenes, no parece ser un obstáculo para la existencia y el éxito de los sellos. Las mujeres actualmente están más integradas en el escenario de la producción editorial, en una relación más simétrica con sus colegas de editoriales independientes, en una economía de colaboración y solidaridad. Pero este es un tema que demanda una investigación detallada que esperamos completar en un futuro próximo. 


\section{REFERENCIAS BIBLIOGRÁFICAS}

Hallewell, Laurence (2005), O livro no Brasil. Sua história, Maria da Penha Villalobos (trad.), São Paulo, Editora da Universidade de São Paulo (Edusp). [1985]

Muzart, Zahidé Lupinacci (2004), "Histórias da Editora Mulheres", Estudos Feministas, 12: 103-105.

Pereira, Alessandra, Gabrielle Freitas, Izabel Fonseca y Letícia Santana (2015), Maria Mazarello Rodrigues, Belo Horizonte, Edição e Ofício.

Ribeiro, Ana Elisa (2017), "Riqueza interdisciplinar e debilidade institucional: Consolidação dos estudos de edição na América Latina. Entrevista de José Luis de Diego", Pontos de Interrogação, 7 (1): 177-186. <https:// www.revistas.uneb. br/index.php/pontosdeint/article/view/3937/2469>

Sapiro, Gisèle (2016), La sociología de la literatura, Laura Fólica (trad.), Buenos Aires, Fondo de Cultura Económica.

Thompson, John B. (2013), Mercadores de cultura. O mercado editorial no século XXI, Alzira Allegro (trad.), São Paulo, Editora da Universidade Estadual Paulista (UNESP). 